\title{
Proton polarisability contribution to the Lamb shift in muonic hydrogen at fourth order in chiral perturbation theory
}

\author{
Michael C. Birse and Judith A. McGovern \\ Theoretical Physics Division, \\ School of Physics and Astronomy, \\ The University of Manchester, \\ Manchester, M13 9PL, UK
}

\begin{abstract}
We calculate the amplitude $T_{1}$ for forward doubly-virtual Compton scattering in heavy-baryon chiral perturbation theory, to fourth order in the chiral expansion and with the leading contribution of the $\gamma \mathrm{N} \Delta$ form factor. This provides a model-independent expression for the amplitude in the low-momentum region, which is the dominant one for its contribution to the Lamb shift. It allows us to significantly reduce the theoretical uncertainty in the proton polarisability contributions to the Lamb shift in muonic hydrogen. We also stress the importance of consistency between the definitions of the Born and structure parts of the amplitude. Our result leaves no room for any effect large enough to explain the discrepancy between proton charge radii as determined from muonic and normal hydrogen.

PACS numbers: 31.30.jr, 12.29.Fe, 13.60.Fz, 14.20.Dh
\end{abstract}

\section{INTRODUCTION}

The recent determination of the proton charge radius from the Lamb shift in muonic hydrogen 1 gives a value that differs by about 5 standard deviations from the CODATA value 2] and from the results of recent electron scattering experiments [3. This has generated a flurry of activity attempting to understand the origin of the discrepancy and whether it could be a signal of physics beyond the Standard Model. Recent reviews of the situation can be found in Refs. [4 7].)

However before concluding that new physics is required, it is essential to examine carefully any possible conventional explanations. As discussed in the reviews, many of the contributions to the Lamb shift are theoretically well constrained. One place where some theoretical uncertainty remains is the contribution of proton structure to two-photon exchange, specifically through the polarisability of the proton.

The energy shift of an $S$-wave hydrogenic state due to two-photon exchange can be expressed in terms of the spin-averaged amplitude for forward doubly-virtual Compton scattering $\left(\mathrm{V}^{2} \mathrm{CS}\right)$ [8]. This comprises two tensor structures:

$$
\begin{aligned}
T^{\mu \nu}= & \left(-g^{\mu \nu}+\frac{q^{\mu} q^{\nu}}{q^{2}}\right) T_{1}\left(\omega, Q^{2}\right) \\
& +\frac{1}{M^{2}}\left(p^{\mu}-\frac{p \cdot q}{q^{2}} q^{\mu}\right)\left(p^{\nu}-\frac{p \cdot q}{q^{2}} q^{\nu}\right) T_{2}\left(\omega, Q^{2}\right),
\end{aligned}
$$

where $p$ and $q$ are the four-momenta of the proton and photon, respectively, $M$ is the nucleon mass, $Q^{2}=-q^{2}$, and $\omega=p \cdot q / M$. Dispersion relations 9, 10, can be used to estimate the amplitudes $T_{1,2}\left(\omega, Q^{2}\right)$ from the corresponding structure functions measured in inelastic electron scattering. These parts of the contribution of proton structure are well determined from the available data, with one important exception: the dispersion relation for $T_{1}$ does not converge and so it requires a subtraction. This is normally done at $\omega=0$, introducing a dependence on the unmeasured amplitude $T_{1}\left(0, Q^{2}\right)$. The slope of this term at $Q^{2}=0$ is given by a low-energy theorem (LET) in terms of the magnetic polarisability of the proton, $\beta$ [11 13. Otherwise its form is unknown and nucleon elastic form-factors are often used to model its $Q^{2}$ dependence [10, 14. The same amplitude is also needed in calculations of the electromagnetic self-energies of the nucleons 15, 16] and it has recently discussed in this context [17.

This approach to determining the amplitude for forward $\mathrm{V}^{2} \mathrm{CS}$ has been questioned in several recent papers. Miller et al. 18, have suggested that off-shell form-factors of the proton could generate new large polarisability contributions to $\mathrm{V}^{2} \mathrm{CS}$. They also questioned the validity of the LET for the slope. Carlson and Vanderhaeghen 19] estimated the $Q^{2}$ dependence from a two-pion exchange model motivated by chiral perturbation theory. They also split the amplitude $T_{1}\left(\omega, Q^{2}\right)$ into nucleon "pole" and "non-pole" terms, in contrast to Pachucki 10, who separated out the full nucleon Born contribution obtained from the Dirac equation with on-shell form factors. Finally, Hill and Paz 20 used an effective field theory, nonrelativistic $\mathrm{QED}$, to calculate $T_{1}\left(0, Q^{2}\right)$, implicitly verifying the LET. They also questioned the use of on-shell form factors to get the $Q^{2}$ dependence of the non-pole Born terms in $T_{1}\left(0, Q^{2}\right)$. They did not attempt to estimate the polarisability contribution but suggested that the uncertainty in two-photon exchange could have been significantly underestimated. More recently, Carlson and Vanderhaeghen 21 have examined the constraints on $T_{1,2}\left(\omega, Q^{2}\right)$ from real Compton scattering and shown that these are incompatible with the model of Miller et al. [18.

To obtain a model-independent result for the form 
of $T_{1}\left(0, Q^{2}\right)$ at small $Q^{2}$, we calculate $T_{1}\left(0, Q^{2}\right)$ within the framework of chiral perturbation theory (ChPT) to fourth order. The polarisability contribution to the Lamb shift has previously been calculated using heavy-baryon chiral perturbation theory (HBChPT) at third order and found to be small [22]. However it is known that fourthorder contributions to the polarisabilities of the proton are larger than naively expected 23. In addition, the $\Delta$ resonance is known to make an important contribution to the magnetic polarisability $\beta[24,26$, and its potential significance for the Lamb shift has been stressed by Pineda [27. We also note that ChPT results for spin-dependent $\mathrm{V}^{2} \mathrm{CS}$ show rapid dependence on $Q^{2}$ at low momenta [28 30]. If the spin-independent amplitude $T_{1}\left(0, Q^{2}\right)$ were to show similar behaviour, this could be important for the Lamb shift. These results imply that it is important to check whether higher-order effects could alter previous conclusions. We do so here using the same $\mathrm{ChPT}$ approach as recently applied to the analysis of real Compton scattering [31, treating nucleons to fourthorder in HBChPT and including effects of the $\Delta$ up to fifth order in the " $\delta$-counting" introduced by Pascalutsa and Phillips [26. This gives us an estimate of the "mass" in the form factor for $T_{1}\left(0, Q^{2}\right)$ which is determined consistently with the value for $\beta$ obtained in Ref. 31].

In view of the questions that have been raised about the LET for $T_{1}\left(0, Q^{2}\right)$, we re-examine its derivation to clarify some of the issues related to it and to the separation of the amplitude into Born and "structure" parts. We also comment on how the LET is embodied in nonrelativistic effective field theories such as HBChPT.

Finally, given the low-energy nature of the theory, it cannot give reliable estimates of the behaviour for larger momenta but we give arguments in favour of a $1 / Q^{2}$ fall-off at large $Q^{2}$, as also obtained from the operatorproduct expansion 32. We use this to estimate the contribution to the Lamb shift and to show that it is too small to explain the deduced discrepancy.

\section{LOW-ENERGY LIMIT}

Before calculating the higher-order contributions of proton structure to $T_{1}$, we first re-examine the low-energy constraints on the proton-structure parts of the amplitude. In the low-momentum limit, the amplitudes for real and virtual Compton scattering can be related through the limited number of tensor structures that respect covariance and gauge invariance, and are even under crossing $\left(q \leftrightarrow-q^{\prime}, \nu \leftrightarrow \mu\right)$. The invariant amplitudes multiplying them should also be free from unphysical kinematic singularities [33, 34] (see also: Refs. [12, 13]). Under these constraints, the spin-independent amplitude can be expanded in terms of five basis tensors:

$$
T^{\mu \nu}=\sum_{i=a, \cdots, e} T_{i}\left(\bar{p}^{2}, q^{2}, q \cdot \bar{p}, q^{\prime} \cdot q\right) t_{i}^{\mu \nu}
$$

where $\bar{p}=\left(p+p^{\prime}\right) / 2$ is the average of the initial and final proton momenta, and we have taken the initial and final photons to have the same virtualities ${ }^{1}$ These tensors may be chosen to be:

$$
\begin{aligned}
t_{a}^{\mu \nu}=-q^{\prime} \cdot q g^{\mu \nu}+q^{\mu} q^{\prime \nu}, \\
t_{b}^{\mu \nu}=q^{\prime} \cdot q \bar{p}^{\mu} \bar{p}^{\nu}-q \cdot \bar{p}\left(\bar{p}^{\mu} q^{\prime \nu}+q^{\mu} \bar{p}^{\nu}\right)+(q \cdot \bar{p})^{2} g^{\mu \nu}, \\
t_{c}^{\mu \nu}=q^{4} g^{\mu \nu}+q^{\prime} \cdot q q^{\prime \mu} q^{\nu}-q^{2}\left(q^{\mu} q^{\nu}+q^{\prime \mu} q^{\prime \nu}\right), \\
t_{d}^{\mu \nu}=q \cdot \bar{p}\left[2 q \cdot \bar{p} q^{2} g^{\mu \nu}-q \cdot \bar{p}\left(q^{\mu} q^{\nu}+q^{\prime \mu} q^{\prime \nu}\right)\right. \\
\left.\quad \quad-q^{2}\left(\bar{p}^{\mu} q^{\prime \nu}+q^{\mu} \bar{p}^{\nu}\right)+q^{\prime} \cdot q\left(\bar{p}^{\mu} q^{\nu}+q^{\prime \mu} \bar{p}^{\nu}\right)\right], \\
t_{e}^{\mu \nu}=q^{4} \bar{p}^{\mu} \bar{p}^{\nu}+(q \cdot \bar{p})^{2} q^{\prime \mu} q^{\nu}-q \cdot \bar{p} q^{2}\left(\bar{p}^{\mu} q^{\nu}+q^{\prime \mu} \bar{p}^{\nu}\right) .
\end{aligned}
$$

Note that only four of the tensors are linearly independent, but there is no way to eliminate any one of them without introducing kinematic singularities in the amplitudes $T_{i}[12,13,34$.

In calculating the two-photon-exchange interaction between a lepton and a proton, it is convenient to split the Compton amplitude into a Born piece, expressed entirely in terms of couplings of a single photon to an on-shell proton, and a "structure" piece involving, for example, the polarisabilities of the proton. Conventionally, the Born amplitude is calculated from a Dirac equation for the proton with Dirac and Pauli form factors, $F_{D}\left(Q^{2}\right)$ and $F_{P}\left(Q^{2}\right)$ respectively (often denoted $F_{1}$ and $F_{2}$ although this invites confusion with inelastic structure functions). The Born contributions to the spin-independent Compton amplitud $\AA^{2}$ obtained in this way have the forms

$$
\begin{aligned}
& T_{a}^{B}=e^{2} \frac{\left(\bar{p}^{2} q^{\prime} \cdot q-(q \cdot \bar{p})^{2}\right) F_{P}\left(2 F_{D}+F_{P}\right)}{M^{3}\left(s-M^{2}\right)\left(u-M^{2}\right)}, \\
& T_{b}^{B}=e^{2} \frac{q^{\prime} \cdot q F_{P}\left(2 F_{D}+F_{P}\right)-4 M^{2} F_{D}^{2}-2 q^{2} F_{D} F_{P}}{M^{3}\left(s-M^{2}\right)\left(u-M^{2}\right)}, \\
& T_{c}^{B}=0, \\
& T_{d}^{B}=-e^{2} \frac{F_{P}\left(F_{D}+F_{P}\right)}{M^{3}\left(s-M^{2}\right)\left(u-M^{2}\right)}, \\
& T_{e}^{B}=0 .
\end{aligned}
$$

As required for the amplitudes corresponding to the tensors of Eq. (3), these have poles only at the on-shell points, $s \equiv(p+q)^{2}=M^{2}$ and $u \equiv\left(p-q^{\prime}\right)^{2}=M^{2}$.

The standard treatment of the Lamb shift [10] subtracts this Born amplitude to leave a residual, structure amplitude that is free of nucleon poles and can be estimated from inclusive-scattering structure functions with

\footnotetext{
${ }^{1}$ If the virtualities are different, there is also a sixth basis tensor. 2 This has been defined by taking a covariant spin average, $T^{\mu \nu}=$ $\operatorname{Tr}\left[\left(\not p^{\prime}+M\right) T_{s}^{\mu \nu}(\not p+M)\right] /\left(8 M^{2}\right)$, similar to that used in Ref. [8]. Up to an overall factor, it corresponds to the spin-independent amplitude in the Breit frame, where there is a unique spin axis defined by the (parallel) initial and final momenta of the proton.
} 
the aid of dispersion relations. This approach has been dismissed by Hill and Paz [20] as "sticking in form factors". However it provides a well-defined expression for the $Q^{2}$ dependence of the Born amplitude and, provided that the non-Born piece is defined consistently with it, there is no problem with this choice. Below, we comment further on the need for consistency between the choice of Born amplitude and the form of the non-Born piece.

First, we look at the LETs satisfied by the non-Born amplitude defined in this way. This amplitude starts at second order in the photon momenta, with terms proportional to $t_{a}$ and $t_{b}$, the only tensors of order $q^{2}$ :

$$
T^{\mu \nu}=T^{B \mu \nu}-4 \pi \beta t_{a}^{\mu \nu}-\frac{4 \pi}{M^{2}}(\alpha+\beta) t_{b}^{\mu \nu}+\mathcal{O}\left(q^{4}\right),
$$

where the proton states are normalised so that the Thomson term is $\left(e^{2} / M\right) \epsilon^{\prime} \cdot \epsilon$. The contributions of proton structure at this order are contained in the two constants multiplying these tensors. They have been expressed in terms of the electric and magnetic polarisabilities of the proton, $\alpha$ and $\beta$, which can be determined from real Compton scattering at low energies. (For details, see the review: Ref. 31.)

In the case of forward $\mathrm{V}^{2} \mathrm{CS}$, the standard amplitudes in Eq. (1) can be related to those multiplying the five tensors of Eq. (3) by

$$
\begin{aligned}
& T_{1}=q^{2} T_{a}-(q \cdot p)^{2} T_{b}-q^{4} T_{c}-2 q^{2}(q \cdot p)^{2} T_{d}, \\
& T_{2}=M^{2}\left(q^{2} T_{b}+q^{4} T_{e}\right) .
\end{aligned}
$$

The residual amplitudes $\bar{T}_{i}=T_{i}-T_{i}^{B}$ satisfy the LETs [8],

$$
\begin{aligned}
& \bar{T}_{1}\left(\omega, Q^{2}\right)=4 \pi Q^{2} \beta+4 \pi \omega^{2}(\alpha+\beta)+\mathcal{O}\left(q^{4}\right), \\
& \bar{T}_{2}\left(\omega, Q^{2}\right)=4 \pi Q^{2}(\alpha+\beta)+\mathcal{O}\left(q^{4}\right) .
\end{aligned}
$$

The LET for $T_{1}\left(0, Q^{2}\right)$ is important in the standard approach to the Lamb shift [10] since the dispersion relation for $\bar{T}_{1}$ requires a subtraction and the LET constrains the low- $Q^{2}$ limit of the subtraction term.

Any introduction of off-shell form factors along the lines suggested by Miller et al. 18] should respect these LETs. The fact that their results for $T_{1}\left(0, Q^{2}\right)$ do not is more than just a numerical inconsistency with real Compton scattering [21, it represents a violation of general principles.

We now return to the question of consistency between Born and non-Born amplitudes. The factors of the Breitframe photon energy $q \cdot \bar{p}$ in the basis tensors of Eq. (3) mean that the Born contributions to the full Compton amplitude $T^{\mu \nu}$ contain pieces without poles corresponding to on-shell intermediate proton states. This can be seen clearly in the case of forward $\mathrm{V}^{2} \mathrm{CS}$, where the Born terms have the forms

$$
T_{1}^{B}\left(\omega, Q^{2}\right)=\frac{e^{2}}{M}\left[\frac{Q^{4}\left(F_{D}\left(Q^{2}\right)+F_{P}\left(Q^{2}\right)\right)^{2}}{Q^{4}-4 M^{2} \omega^{2}}-F_{D}\left(Q^{2}\right)^{2}\right],
$$

$$
\begin{aligned}
T_{2}^{B}\left(\omega, Q^{2}\right)= & \frac{4 e^{2} M Q^{2}}{Q^{4}-4 M^{2} \omega^{2}} \\
& \times\left[F_{D}\left(Q^{2}\right)^{2}+\left(Q^{2} / 4 M^{2}\right) F_{P}\left(Q^{2}\right)^{2}\right]
\end{aligned}
$$

and the second piece of $T_{1}^{B}$ contains no poles at $\omega=$ $\pm Q^{2} / 2 M$.

Various authors (for recent examples, see Refs. 17, 19, 20]) have suggested that, rather than splitting the Compton amplitude into "Born" and "structure" pieces, it is better separate it into "pole" and "non-pole" pieces. In that approach, the second term of Eq. (9) would count as part of the non-pole amplitude, along with the polarisabilities and other structure contributions. However, as shown by Walker-Loud et al. [17, this separation is not unique since the form of the pole piece depends on the choice of basis tensors. In the basis of Eq. (3) above, the $F_{D}\left(Q^{2}\right)^{2}$ term arises from a pole term in the amplitude $T_{b}$ and, furthermore, it is required to keep the full Born amplitude free from kinematic singularities at $Q^{2}=0$. All of this implies that the separation into Born plus structure is the more natural one.

The role of this term can be seen more clearly by expanding it in powers of $Q^{2}$ to get

$$
F_{D}\left(Q^{2}\right)^{2}=1-\left[\frac{1}{3} r_{c}^{2}-\frac{\kappa}{2 M^{2}}\right] Q^{2}+\mathcal{O}\left(Q^{4}\right),
$$

where $r_{c}$ is the charge radius of the proton (defined in terms of the slope of the Sachs electric form factor) and $\kappa=F_{P}(0)$ is its anomalous magnetic moment. This shows that the leading term in this piece of $T_{1}^{B}$ gives rise to a term proportional to $q_{\mu} q_{\nu} / q^{2}$ in the scattering amplitude. In the full amplitude this cancels against a similar singular term arising from $T_{2}^{B}$.

The next term in $F_{D}\left(Q^{2}\right)^{2}$, of order $Q^{2}$, generates a contribution to the scattering amplitude that remains finite for real photons, $Q^{2} \rightarrow 0$. Shifting this contribution from the Born amplitude to the structure part would alter the coefficient of $Q^{2}$ in $\bar{T}_{1}$ and so would correspond to a definition of the magnetic polarisability $\beta$ that differed from the conventional one. Carlson and Vanderhaeghen [19] keep only the pole pieces of the Born amplitude but the form they take for $\bar{T}_{1}\left(0, Q^{2}\right)$ is inconsistent with this since it satisfies an LET with conventional definition of $\beta$. The fact that the order- $Q^{2}$ term in $T_{1}\left(0, Q^{2}\right)$ contains this piece from the expansion of $F_{D}\left(Q^{2}\right)^{2}$ leads WalkerLoud et al. [17] to use the same definition for the structure part of the $\mathrm{V}^{2} \mathrm{CS}$ amplitude as we advocate, albeit without the explicit justification presented here.

In the context of the HBChPT calculation described in the next section, consistency between the Born and structure amplitudes as usually defined means that we must subtract the full Born amplitude of Eq. (9) expanded to the appropriate order in $Q^{2}$. Specifically, in addition to all pole terms, we need to subtract the terms in the expansion of $F_{D}\left(Q^{2}\right)^{2}$. To do this we need the Dirac form 
factor calculated to third order in the chiral expansion, which is given in Ref. 35.

The LETs of Eqs. (7, 8) are built into the effective field theory for a nonrelativistic particle through the constraints of relativity, often expressed in terms of "reparametrisation invariance" [36]. The Lagrangian of any such theory contain three types of structure with two derivatives of the photon fields [37, 38]. Two of these contribute to the polarisabilities $\alpha+\beta$ and $\beta$. In contrast, the third has a coefficient that is fixed in terms of lowerorder constants that correspond to the charge radius and anomalous magnetic moment. It generates the order- $Q^{2}$ term of the non-pole piece of $T_{1}^{B}$ discussed above. This is illustrated by the amplitude calculated in nonrelativistic QED, Eq. (11) of Ref. 20, which contains both the nonpole Born piece arising from the second term of Eq. (11) and the magnetic polarisability.

\section{CHIRAL PERTURBATION THEORY}

The specific amplitude we consider here is $T_{1}\left(0, Q^{2}\right)$ for forward $\mathrm{V}^{2} \mathrm{CS}$, which appears as the subtraction term in the dispersion relation for $T_{1}\left(\omega, Q^{2}\right)$ [10] and so forms one of the least-well determined contributions of proton structure to the Lamb shift 18 20. Its leading term, of order $Q^{2}$, is fixed by the LET discussed in the previous section but contributions from its higher-order terms vanish in the real-photon limit and so are subject to no such constraints. If these terms were to grow rapidly with $Q^{2}$, they could lead to unexpectedly large structure contributions to the Lamb shift. They have been calculated by Nevado and Pineda 22] at third-order where, like the polarisabilities [35, 39, they are predictions that do not require any two-photon low-energy constants. However there are large contributions at fourth-order to both spinindependent and spin-dependent real Compton scattering [23, 28, 30]. In addition, the effects of $\Delta$ resonance are important for the magnetic polarisability and could also play a significant role in the Lamb shift 27. To examine whether these effects could be similarly important for the Lamb shift, we have calculated the $Q^{4}$ piece of forward $\mathrm{V}^{2} \mathrm{CS}$ treating nucleons to fourth order in HBChPT. We also include the leading contribution of the $\gamma \mathrm{N} \Delta$ form factor, a term of fifth order in the $\delta$ counting of Ref. [26].

The calculation of $T_{1}\left(0, Q^{2}\right)$ has a very close correspondence to that of forward real Compton scattering [35, 39, 40]. To take advantage of this, we choose a gauge in which the polarisation vectors of the virtual photons are purely space-like (in the proton rest frame), so that the conditions $p \cdot \epsilon=0, q \cdot \epsilon=0$ which were used in the real case still hold. With this choice, the diagrams that contribute are the same, and in many cases the resulting expressions can be obtained from the corresponding ones for real photons with the aid of some straightfoward substitutions of kinematic variables. A representative subset of the fourth-order nucleon diagrams is shown in Fig. 1; the full set is given in Fig. 1 of Ref. [40. (The third-order diagrams can be found in Fig. 1 of Ref. [22.)

To determine the "structure" piece of the amplitude, $\bar{T}_{1}$, we need to separate it from the Born terms. This separation is somewhat subtle in $\mathrm{HBChPT}$, and cannot just be done on a diagram by diagram basis [40. Some diagrams, such as Fig. 1(a), can immediately be identified as parts of the "magnetic" Born term (the first, "pole" term of Eq. (9p). However there is also a one-nucleon-reducible diagram in which one photon couples to the nucleon via a magnetic-moment coupling and the other via a pion loop, Fig. 1(c). As discussed in Ref. 41, this contributes to both the magnetic Born and structure pieces of real Compton scattering. The structure part arises from the energy dependence of the pion loop which corresponds to an "off-shell form factor" of the sort proposed by Miller et al. [18, but with a calculable form. This illustrates the fact that such off-shell dependences cannot be distinguished from structure effects such as polarisabilities.

In the case of space-like virtual photons considered here, particular care is needed with the one-nucleon reducible diagrams since the the leading propagator for the intermediate nucleon is $2 M / Q^{2}$. This means that such diagrams contribute at one order lower than in the expansion of real Compton scattering. For example, the diagram Fig. 1(c) just discussed contributes at third order, where it forms part of the magnetic Born piece of $T_{1}$. At fourth order there is a very similar graph but with a second-order insertion in the nucleon propagator within the loop. This contributes to both Born and "structure" pieces of the amplitude. To this order, all other reducible diagrams with the enhanced $2 M / Q^{2}$ propagator (diagrams like Fig. 1(a) but with higher-order vertices) contribute only to the magnetic Born term.

The sum of all graphs (at third and fourth orders) has no constant term; the coefficient of $Q^{2}$ is the same as the loop contribution to $\beta[23$ plus a piece proportional to the third-order loop contribution to the derivative of the single-photon form-factor $F_{D}$. The divergences in these loop integrals are renormalised by photon-nucleon seagulls, Fig. 1(e), arising from terms in the fourth-order chiral Lagrangian. These include a term with the structure $F_{\mu \nu} F^{\mu \nu}$ which is the same one that appears in the renormalisation of the magnetic polarisability $\beta$. This term is a combination of $\widehat{O}_{89,91,93,118}^{(4)}$ in the minimal Lagrangian of Fettes et al. 38. That Lagrangian also contains terms with the structure $v^{\mu} v^{\nu} F_{\lambda \mu} F_{\nu}^{\lambda}$ which contribute to $\alpha+\beta$ and so are not relevant to forward $\mathrm{V}^{2} \mathrm{CS}$.

In addition, the fourth-order heavy-baryon effective Lagrangian contains terms with coefficients that are fixed by relativistic invariance in terms of coefficients from the lower-order Lagrangians. A combination of two of these is needed here, $X_{40}$ and $X_{52}$ of Ref. 38. These have the structure $\left[D_{\mu}, F^{\mu \lambda}\right] D_{\lambda}+$ h.c., which does not correspond to either of the polarisabilities of the nucleon. But, like the similar $c_{M}$ term in the norelativistic QED Lagrangian of Manohar [37], the coefficient of this is given in terms of the second-order anomalous magnetic moment of the proton and the third-order coefficient that contributes to 

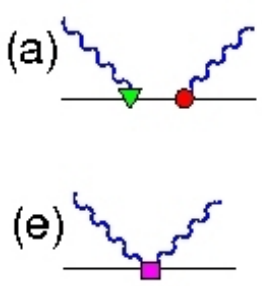

(b)

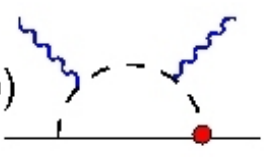

(f)

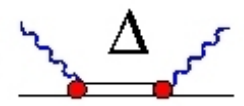

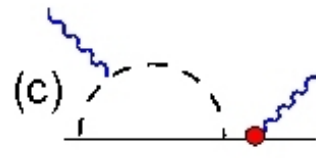

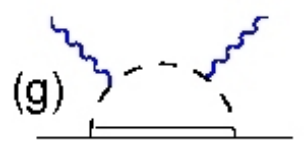

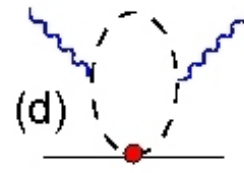

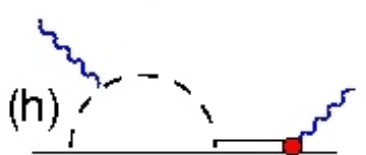

FIG. 1. Some diagrams contributing to Compton scattering in HBChPT: (a) a one-nucleon-reducible tree graph; (b-d) a representative selection of $\pi \mathrm{N}$ loop graphs that contribute at fourth order; (e) the fourth-order seagull contribution to $\beta$; (f, g) $\Delta$ contributions at order $\delta^{3}$ and (h) at $\delta^{5}$. In these graphs the circle, triangle and square denote vertices from the second-, third- and fourth-order Lagrangians, respectively.

the charge radius. In the present context, this seagull diagram renormalises the loop contributions of order $Q^{2}$ to $F_{D}\left(Q^{2}\right)^{2}$, the "non-pole" Born term.

As well as fourth-order terms in the chiral expansion, we have also examined the effect of explicitly including the $\Delta$ resonance since this low-lying excitation can play an important role in the responses of a nucleon to external fields, particularly in the magnetic polarisability 24 26]. Working within the $\delta$ expansion introduced by Pascalutsa and Phillips [26, we find that the leading $\left(\right.$ order $\left.-\delta^{4}\right) \Delta$-pole graph, Fig. 1(f), has the same form as the seagull contribution to $\beta$ and so can simply be absorbed in the corresponding low-energy constant. In a similar way, the leading $\pi \Delta$ loops such as Fig. 1(g) can to a good approximation be absorbed into the the values of the low-energy constants appearing in the fourth-order tadpole diagrams, Fig. 1(d). The full expression differs from the pieces already included in the fourth-order result by about $16 \%$, which is well within the uncertainty introduced by the rather poorly-known second-order constants, and so we do not give it explicitly.

The one $\Delta$-pole contribution we do include arises from Fig. 1(h), where the $\pi \mathrm{N}$ loop generates a $\gamma \mathrm{N} \Delta$ form factor. This diagram is of fifth order in $\delta$, which is higher than the other terms considered here, but it provides the leading contribution of this form factor to $T_{1}\left(0, Q^{2}\right)$ at order $Q^{4}$. We have therefore included it as an estimate of the likely influence of the $\Delta$.

After subtracting $T_{1}^{B}$ as discussed above, we arrive at the following result for $\bar{T}_{1}$ :

$$
\begin{aligned}
& \bar{T}_{1}\left(0, Q^{2}\right) \\
& =4 \pi \beta Q^{2}-\left[\frac{e^{2} g_{A}^{2}}{1280 \pi f_{\pi}^{2} m_{\pi}^{3}}\right. \\
& \quad+\frac{e^{2}}{480 \pi^{2} f_{\pi}^{2} m_{\pi}^{2}}\left(4 c_{1}+c_{2}-2 c_{3}-\frac{g_{A}^{2}}{M_{\mathrm{N}}}\left(4+5 \mu_{s}\right)\right) \\
& \left.\quad+\frac{e^{2} g_{M} g_{A} g_{\pi \mathrm{N} \Delta}}{144 \pi f_{\pi}^{2} m_{\pi}\left(M_{\Delta}^{2}-M_{\mathrm{N}}^{2}\right)}\right] Q^{4}+\mathcal{O}\left(Q^{6}\right) .
\end{aligned}
$$

Full expressions for the contributions to the amplitude, not expanded in powers of $Q^{2}$, are given in the Appendix.
In evaluating this numerically, we take $g_{A}=1.27, f_{\pi}=$ $92.2 \mathrm{MeV}$, and $\mu_{s}=0.88$. The constants $c_{i}$ from the second-order Lagrangian have been determined from $\pi \mathrm{N}$ scattering and the resulting values, as quoted by Bernard [44, are $c_{1}=-0.9_{-0.5}^{+0.2}, c_{2}=3.3 \pm 0.2, c_{3}=-4.7_{-1.0}^{+1.2}$, all in $\mathrm{GeV}^{-1}$. However a more tightly constrained value of $c_{3}=-4.78 \pm 0.2 \mathrm{GeV}^{-1}$ has been obtained from $p p$ scatttering [45], and we use this here.

For the couplings of the $\Delta$, we take the values obtained by Pascalutsa et al. 42, 43] of $g_{M}=2.9$ for the magnetic $\gamma \mathrm{N} \Delta$ coupling constant and $g_{\pi \mathrm{N} \Delta}=1.425$. These couplings provide a good description of real Compton scattering below the $\Delta$ peak [31] but they do depend on the choice of Lagrangian. Nonetheless a very similar value for the product $g_{A} g_{\pi \mathrm{N} \Delta}$ has also been found to give a good description of Compton scattering within a purely nonrelativistic framework for the $\Delta$ [46], despite $\sim 30 \%$ differences in the separate couplings. A more serious source of uncertainty is the fact that these analyses are mainly sensitive to the couplings close to the $\Delta$ peak, whereas we need the values at zero energy. To account for this we allow for a $20 \%$ error on $g_{A} g_{\pi \mathrm{N} \Delta}$

If we express our result in the form of a form factor,

$$
\bar{T}_{1}\left(0, Q^{2}\right) \simeq 4 \pi \beta Q^{2}\left(1-\frac{Q^{2}}{M_{\beta}^{2}}+\mathcal{O}\left(Q^{4}\right)\right),
$$

its slope is given by

$$
M_{\beta}^{2}=\frac{\beta}{3 \times 10^{-4} \mathrm{fm}^{3}}(455 \pm 32 \mathrm{MeV})^{2} .
$$

The magnetic polarisability $\beta$ has recently been determined from a fit to real Compton scattering within the same framework as used here, giving $\beta=(3.1 \pm$ $0.5) \times 10^{-4} \mathrm{fm}^{3}$ (including the statistical and Baldin Sum Rule errors only) 31. Using this and the values for the $c_{i}$ and $\Delta$ couplings above, with their errors, gives $M_{\beta}=460 \pm 50 \mathrm{MeV}$ for the mass parameter in $\bar{T}_{1}\left(0, Q^{2}\right)$. This has a size $\sim 3 m_{\pi}$, which is "natural" for an effect with important contributions from pion loops.

Without the fourth-order and $\Delta$ contributions to the slope, $455 \mathrm{MeV}$ would be replaced by $588 \mathrm{MeV}$ in 
Eq. (14). In view of this change from third to fourth order, the residual error due to neglect of higher-order terms is expected to be comparable to the uncertainty arising from the low-energy constants. However the $\Delta$ contributions are likely to provide a larger source of uncertainty as the expansion parameter in the $\delta$ expansion is not particularly small. If we triple the error associated with of the order- $Q^{4}$ term in Eq. 12 to take account of these uncertainties, then we get

$$
M_{\beta}=460 \pm 100 \pm 40 \mathrm{MeV},
$$

where the first error is from the order- $Q^{4}$ term and the second is due to $\beta$.

\section{LAMB SHIFT}

The calculation outlined in the previous section leads to a model-independent result for the $\mathrm{V}^{2} \mathrm{CS}$ amplitude $\bar{T}_{1}\left(0, Q^{2}\right)$ up to order $Q^{4}$. This constrains the subtraction term in the dispersion relation for $\bar{T}_{1}\left(\omega, Q^{2}\right)$ in a way that does not rely on the ad hoc use of the magnetic form factor of the proton as in Ref. [10. However it is not sufficient to estimate the polarisability contribution to the muonic Lamb shift, since that requires an integral over $Q^{2}$.

Our chiral calculation gives an expression for $\bar{T}_{1}\left(0, Q^{2}\right)$ as a function of $Q^{2}$ which can be found in the Appendix. However, this cannot be used for large $Q^{2}$ since it contains terms (such as the fourth-order seagull) that do not vanish as $Q^{2} \rightarrow \infty$. Such terms were not present in the third-order calculation of Nevado and Pineda [22, where the only photon-nucleon seagull is the one that gives the Thomson limit and so is part of the Born amplitude.

Other information on the form of $T_{1}\left(0, Q^{2}\right)$ comes from the large- $Q^{2}$ limit where the operator product expansion leads to $1 / Q^{2}$ for the dominant behaviour 32 (as noted in Ref. [20]). This form can also be understood from the quark counting rules that apply to the partonic regime 47, 48. In this regime there is no single-quark contribution to $T_{1}\left(0, Q^{2}\right)$, as can be seen from the exact cancellation of the two terms in Eq. (9) at $\omega=0$ for pointlike fermions. Instead one-gluon exchange between two quarks gives the dominant contribution and the counting rules show that this falls off like $1 / Q^{2}$.

Here we estimate the contribution to the Lamb shift by matching our result smoothly onto the expected high- $Q^{2}$ behaviour. To do this we write

$$
\bar{T}_{1}\left(0, Q^{2}\right)=4 \pi \beta Q^{2} F_{\beta}\left(Q^{2}\right),
$$

and take the dipole form

$$
F_{\beta}\left(Q^{2}\right)=\frac{1}{\left(1+Q^{2} / 2 \bar{M}_{\beta}^{2}\right)^{2}},
$$

for the form factor. This matching could be carried out at $Q^{2}=0$, in which case $\bar{M}_{\beta}$ is just the value $M_{\beta}$ determined from the slope of the ChPT form factor in the previous section. However nonanalytic terms arising from pion loops can lead to non-dipole dependences of form factors on $Q^{2}$ in the region $Q^{2} \lesssim\left(3 m_{\pi}\right)^{2}$ [49, 50]. In Fig. 2 we show the full ChPT result for the form factor $F_{\beta}\left(Q^{2}\right)$ compared to dipole forms with masses $\bar{M}_{\beta}=460$ and $510 \mathrm{MeV}$. We see that the full form factor and the extrapolation from $Q^{2}=0$ do deviate for $Q^{2} \gtrsim 0.03 \mathrm{GeV}^{2}$, although the differences lie well within the uncertainties of our calculation. In fact the $\mathrm{ChPT}$ result in the region from $Q^{2} \simeq 0.1$ to nearly $0.3 \mathrm{GeV}^{2}$ has a form that is very similar to a dipole with a mass of about $510 \mathrm{MeV}$. If we match onto the dipole form anywhere in this region we get a mass close to this value. In estimating the contribution to the Lamb shift we have therefore used Eq. (17) with

$$
\bar{M}_{\beta}=485 \pm 100 \pm 40 \pm 25 \mathrm{MeV},
$$

where the first two errors arise from the order- $Q^{4}$ term and $\beta$, as discussed above, and the last is from the choice of matching point for the extrapolation. The shaded region in Fig. 2 corresponds to our estimate of the the uncertainties associated with our calculation of the order$Q^{4}$ term by showing dipole forms with masses in the range 385 to $585 \mathrm{MeV}$.

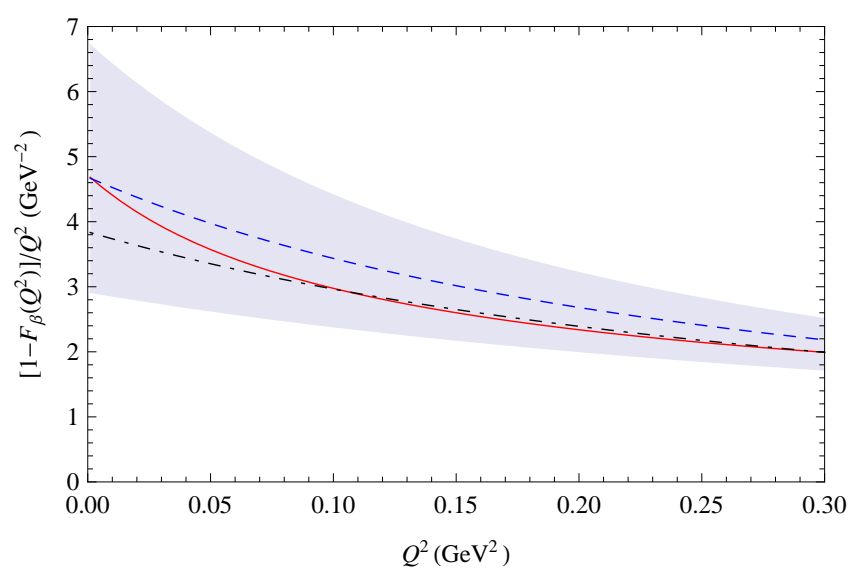

FIG. 2. The slope of the form factor for $\mathrm{V}^{2} \mathrm{CS}$, plotted in the form $\left[1-F_{\beta}\left(Q^{2}\right)\right] / Q^{2}$ to emphasise variations at small $Q^{2}$. The solid (red) curve shows the full result of ChPT calculation. The dashed (blue) curve is the dipole form of Eq. (17) fitted to the slope at $Q^{2}=0$. The dot-dashed (black) curve is a dipole with a mass $\bar{M}_{\beta}=510 \mathrm{MeV}$. The shaded region shows dipole forms with masses in range 385 to $585 \mathrm{MeV}$.

The contribution of the subtraction term to the Lamb 
shift is, from Eq. (34) of Ref. [10, $]^{3}$

$$
\begin{aligned}
\Delta E_{\mathrm{sub}}=\frac{\alpha_{\mathrm{EM}} \phi(0)^{2}}{4 \pi} \int_{0}^{\infty} \mathrm{d} Q^{2} \frac{\bar{T}_{1}\left(0, Q^{2}\right)}{Q^{2}} \\
\times\left[1+\left(1-\frac{Q^{2}}{2 m^{2}}\right)\left(\sqrt{\frac{4 m^{2}}{Q^{2}}+1}-1\right)\right],
\end{aligned}
$$

where $m$ is the lepton mass and

$$
\phi(0)^{2}=\frac{1}{8 \pi}\left(\frac{\alpha_{\mathrm{EM}} m M_{p}}{m+M_{p}}\right)^{3}
$$

is the square of the $2 S$ wave function at the origin. Although we evaluate this taking the dipole form of Eq. (17) for all $Q^{2}$, we have also checked that its integral for $\bar{M}_{\beta} \simeq 510 \mathrm{MeV}$ agrees to better than $1 \%$ with that of the full form matched onto a dipole at $Q^{2}=0.2 \mathrm{GeV}^{2}$. Note that the leptonic factor strongly weights the low- $Q^{2}$ region of this integral, with $90 \%$ coming from the region $Q^{2} \leq 0.3 \mathrm{GeV}^{2}$. This means that matching onto the correct form for larger values of $Q^{2}$ is not crucial, provided that the assumed form does not have a much longer tail than the dipole.

Evaluating the integral using Eq. 17] for $\bar{T}_{1}\left(0, Q^{2}\right)$ we get

$$
\Delta E_{\mathrm{sub}}=4.2 \pm 1.0 \mu \mathrm{eV}
$$

for the contribution to the Lamb shift in muonic hydrogen. The error here includes our estimate of the uncertainties associated with higher-order effects, as discussed in the previous section. However we note that largest single uncertainty comes from the value for $\beta$, which appears both as an overall factor in $\bar{T}_{1}\left(0, Q^{2}\right)$ and in the mass parameter $M_{\beta}$, as given by Eq. (14).

Our result for $\Delta E_{\text {sub }}$ is more than twice that obtained by Pachucki [10. Most of the difference is due to the modern value for $\beta$, which is about twice the one used in that work. There is also a further $\sim 10 \%$ increase from the use of the form factor with the correct asymptotic form. Our result is smaller that the one of Carlson and Vanderhaeghen [19] who used a similar value for $\beta$ as ours but whose model form factor leads to $T_{1}\left(0, Q^{2}\right)$ growing like $\ln Q^{2}$ asymptotically.

Finally, to estimate the total two-photon exchange contribution to the Lamb shift, we add in the "elastic" (Born) and dispersive contributions from Pachucki's treatment [10, 51. We use the updated numerical results from Carlson and Vanderhaeghen [19] and, for consistency with the definition of $\beta$, we reinstate the "nonpole" Born piece. The latter gives about $4.8 \mu \mathrm{eV}$ for

\footnotetext{
${ }^{3}$ Note that our definition of $T_{1}$ differs from that of Ref. [10] by a factor of $e^{2} M$.
}

the form factor of Ref. [52] and so is comparable in importance to the subtraction term. The main sources of uncertainty are $\beta$ in our subtraction term above and the dependence of the elastic contribution on the choice of parametrisation of the proton form factors (which we take as $\pm 1.6 \mu \mathrm{eV}$, based on the results in Ref. [19]). The bottom line for the energy is

$$
\Delta E_{2 \gamma}=-33 \pm 2 \mu \mathrm{eV},
$$

which is not significantly different from the values of about $-35 \mu \mathrm{eV}$ obtained in Refs. 10, 14 but which does not rely on a model for the form factor in $\bar{T}_{1}\left(0, Q^{2}\right)$.

\section{SUMMARY}

In this work, we have calculated the $\mathrm{V}^{2} \mathrm{CS}$ amplitude $T_{1}\left(0, Q^{2}\right)$ at fourth order in HBChPT, and including contributions of the $\Delta$ resonance. Our result provides a model-independent constraint on the low-momentum form of the structure part of this amplitude. Since the contribution of this amplitude to the Lamb shift is dominated by the low-momentum region, this can significantly reduce the theoretical uncertainty in contribution of the polarisability of the proton to the Lamb shift, which has been the subject of some recent debate [18 21].

When our amplitude is matched smoothly onto the high- $Q^{2}$ behaviour expected in the partonic regime [20, we obtain a contribution to the Lamb shift that, perhaps unsurprisingly, is similar in magnitude to previous, more model-dependent determinations [10, 19].

In reaching our result we have re-examined the LETs for $\mathrm{V}^{2} \mathrm{CS}$ and how they are embodied in effective field theories. The constraints of covariance, gauge invariance and crossing symmetry as well as lack of kinematic singularities mean that the only structure terms of order $Q^{2}$ are given by the electric and magnetic polarisabilities. This is reflected by the appearance in fourth-order effective Lagrangians of only two types of seagull term with adjustable coefficients, corresponding to the two polarisabilities. These Lagrangians do contain other seagull terms at this order, but these all have coefficents that are fixed by the requirements of covariance. In particular, there is one that contributes to $T_{1}\left(0, Q^{2}\right)$ at order $Q^{2}$, but only through the "non-pole" piece of the Born amplitude. It is important keep this term in the Born amplitude, rather than subsuming it into the structure part [19, as otherwise the magnetic polarisability in the LET Eq. (7) will not correspond to its conventional definition.

Our results leave no room for any large additional polarisability effect arising from off-shell form factors, as suggested by Miller et al. [18. The form factor appearing in $\bar{T}_{1}\left(0, Q^{2}\right)$ falls off with $Q^{2}$ and its mass parameter has a natural size for a pion-loop effect. We see no sign of any rapid growth at low $Q^{2}$ that could lead to a large contribution to the Lamb shift. 
There is thus no evidence that effects of proton polarisability can explain the difference between the proton charge radius as recently determined from the muonic Lamb shift [1] and that obtained from other experiments [2, 3]. The resolution of this puzzle must lie elsewhere, perhaps in re-analyses of the older experiments as suggested by Lorenz et al. 55.

\section{ACKNOWLEDGMENTS}

We are grateful to G. Miller for discussions that prompted this work. This work was supported by the UK STFC under grants ST/F012047/1 and ST/J000159/1.

\section{Appendix: Full amplitude}

Our result for the the $\mathrm{V}^{2} \mathrm{CS}$ amplitude as a full function of $Q^{2}$ can be expressed in the form

$$
\bar{T}_{1}=\bar{T}_{1}^{(3)}+\bar{T}_{1}^{(4)}+\bar{T}_{1}^{(\Delta)},
$$

where the third-order, fourth-order and $\Delta$-pole contributions are:

$$
\begin{aligned}
\bar{T}_{1}^{(3)}= & \frac{e^{2} g_{A}^{2} m_{\pi}}{16 \pi f_{\pi}^{2}}\left[1-\frac{2}{\sqrt{Q^{2}}} \tan ^{-1}\left(\frac{\sqrt{Q^{2}}}{2}\right)\right]=4 \pi \frac{e^{2} g_{A}^{2}}{768 \pi^{2} m_{\pi} f_{\pi}^{2}} m_{\pi}^{2} Q^{2}+\mathcal{O}\left(Q^{4}\right), \\
\bar{T}_{1}^{(4)}= & 4 \pi \delta \beta m_{\pi}^{2} Q^{2} \\
& +\frac{e^{2} m_{\pi}^{2}}{288 \pi^{2} f_{\pi}^{2}}\left\{12\left(c_{3}-2 c_{1}\right)\left(Q^{2}+12\right)+16 c_{2}\left(Q^{2}+3\right)+3 \frac{g_{A}^{2}}{M_{\mathrm{N}}}\left(6-\left(12 \mu_{s}+13\right) Q^{2}\right)\right. \\
& -\frac{12}{\sqrt{Q^{2}\left(Q^{2}+4\right)}} \tanh ^{-1}\left(\sqrt{\frac{Q^{2}}{Q^{2}+4}}\right)\left[12\left(c_{3}-2 c_{1}\right)\left(Q^{2}+4\right)+c_{2}\left(Q^{2}+4\right)^{2}\right. \\
\bar{T}_{1}^{(\Delta)}= & \left.\frac{2 e^{2}}{\left(M_{\Delta}^{2}-M_{\mathrm{N}}^{2}\right)\left(M_{\Delta}+M_{\mathrm{N}}\right)} m_{\pi}^{2} Q^{2}\left\{g_{M}^{2}+g_{M} \frac{g_{A}^{2}}{M_{\mathrm{N}}}\left(\left(\mu_{s}+1\right) Q^{2}\left(Q^{2}+4\right)-2\right)\right]\right\} \\
96 \pi f_{\pi}^{2} \sqrt{Q^{2}} & (\mathrm{~A} .3) \\
&
\end{aligned}
$$

where $Q^{2}$ has been expressed in units of $m_{\pi}^{2}$. Here $\delta \beta$ denotes the sum of fourth-order loop and counterterm contributions to $\beta$. The subsequent terms in $\bar{T}_{1}^{(4)}$ are of order $Q^{4}$ and higher, and hence contribute to the form factor that is needed for the Lamb shift. The value of $\delta \beta$ is independent of the choice of renormalisation scale and it is adjusted so that the complete term proportional to $Q^{2}$ in $\bar{T}_{1}$ gives the observed magnetic polarisability, as determined by a fit to Compton scattering data in Ref. [31].
[1] R. Pohl et al., Nature 466, 213 (2010).

[2] P. J. Mohr, B. N. Taylor and D. B. Newell, Rev. Mod. Phys. 80, 633 (2008) arXiv:0801.0028.

[3] J. C. Bernauer et al. (A1 Collaboration), Phys. Rev. Lett. 105, 242001 (2010) arXiv:1007.5076.

[4] U. D. Jentschura, Ann. Phys. 326, 500 (2011) arXiv:1011.5275.
[5] U. D. Jentschura, Ann. Phys. 326, 516 (2011) arXiv:1011.5453.

[6] E. Borie, Ann. Phys. 327, 733 (2012) arXiv:1103.1772.

[7] P. J. Mohr, B. N. Taylor and D. B. Newell, arXiv:1203.5425

[8] J. Bernabéu and R. Tarrach, Ann. Phys. 102, 323 (1976) 
[9] J. Bernabéu and C. Jarlskog, Nucl. Phys. B 60, 347 (1973).

[10] K. Pachucki, Phys. Rev. A 60, 3593 (1999) arXiv:physics/9906002.

[11] S. Scherer, A. Yu. Korchin and J. H. Koch, Phys. rev. C 54, 904 (1996) arXiv:nucl-th/9605030.

[12] D. Drechsel, G. Knoechlein, A. Metz and S. Scherer, Phys. Rev. C 55, 424 (1997) arXiv:nucl-th/9608061.

[13] H. W. Fearing and S. Scherer, Few-Body Syst. 23, 111 (1998) arXiv:nucl-th/9607056.

[14] A. P. Martynenko, Phys. Atom. Nucl. 69, 1309 (2006) arXiv:hep-ph/0509236.

[15] A. Zee, Phys. Rept. 3, 127 (1972)

[16] J. Gasser and H. Leutwyler, Phys. Rept. 87, 77 (1982).

[17] A. Walker-Loud, C. E. Carlson and G. A. Miller, arXiv:1203.0254.

[18] G. A. Miller, A. W. Thomas, J. D. Carroll and J. Rafelski, Phys. Rev. A 84, 020101 (2011) arXiv:1101.4073.

[19] C. E. Carlson and M. Vanderhaeghen, Phys. Rev. A 84, 020102 (2011) arXiv:1101.5965.

[20] R. J. Hill and G. Paz, Phys. Rev. Lett. 107, 160402 (2011) arXiv:1103.4617.

[21] C. E. Carlson and M. Vanderhaeghen, arXiv:1109.3779.

[22] D. Nevado and A. Pineda, Phys. Rev. C 77, 035202 (2008) arXiv:0712.1294.

[23] V. Bernard, N. Kaiser, A. Schmidt and U.-G. Meissner, Phys. Lett. B 319, 269 (1993) arXiv:hep-ph/9309211; V. Bernard, N. Kaiser, U.-G. Meissner and A. Schmidt, Z. Phys. A 348, 317 (1994) arXiv:hep-ph/9311354.

[24] M. N. Butler and M. J. Savage, Phys. Lett. 294, 369 (1992) arXiv:hep-ph/9209204.

[25] T. R. Hemmert, B. R. Holstein and J. Kambor, Phys. Rev. D 55, 5598 (1997) arXiv:hep-ph/9612374.

[26] V. Pascalutsa and D. R. Phillips, Phys. Rev. C 67, 055202 (2003) arXiv:nucl-th/0212024.

[27] A. Pineda, Phys. Rev. C 71, 065205 (2005) arXiv:hep$\mathrm{ph} / 0412142$.

[28] V. Bernard, N. Kaiser and U.-G. Meissner, Phys. Rev. D 48, 3062 (1993) arXiv:hep-ph/9212257.

[29] X. Ji, C.-W. Kao and J. Osborne, Phys. Lett. B 472, 1 (2000) arXiv:hep-ph/9910256.

[30] V. Bernard, T. R. Hemmert and U.-G. Meissner, Phys. Lett. B 545, 105 (2002) arXiv:hep-ph/0203167; Phys. Rev. D 67, 076008 (2003) arXiv:hep-ph/0212033.

[31] H. W. Griesshammer, J. A. McGovern, D. R. Phillips and G. Feldman, Prog. Part. Nucl. Phys. 67, 841 (2012)
arXiv:1203.6834

[32] J. C. Collins, Nucl. Phys. B 149, 90 (1979).

[33] W. A. Bardeen and W.-K. Tung, Phys. Rev. 173, 1423 (1968).

[34] R. Tarrach, Nuovo Cim, 28A, 409 (1975).

[35] V. Bernard, N. Kaiser and U.-G. Meissner, Int. J. Mod. Phys. E 4, 193 (1995).

[36] M. E. Luke and A. V. Manohar, Phys. Lett. B 286, 348 (1992) arXiv:hep-ph/9205228.

[37] A. V. Manohar, Phys. Rev. D 56, 230 (1997) arXiv:hep$\mathrm{ph} / 9701294$.

[38] N. Fettes, U.-G. Meissner, M. Mojzis and S. Steininger, Ann. Phys. 283, 273 (2000); erratum ibid. 288, 249 (2001).

[39] V. Bernard, N. Kaiser and U.-G. Meissner, Nucl. Phys. B 373, 346 (1992).

[40] J. A. McGovern, Phys. Rev. C 63, 064608 (2001); erratum ibid. 66, 039902 (2002) arXiv:nucl-th/0101057.

[41] M. C. Birse, X. Ji and J. A. McGovern Phys. Rev. Lett. 86, 3204 (2001) arXiv:nucl-th/0011054.

[42] V. Pascalutsa and M. Vanderhaeghen, Phys. Rev. D 73, 034003 (2006) arXiv:hep-ph/0512244.

[43] V. Pascalutsa, M. Vanderhaeghen and S. N. Yang, Phys. Rept. 437, 125 (2007) arXiv:hep-ph/0609004.

[44] V. Bernard, Prog. Part. Nucl. Phys. 60, 82 (2008) arXiv:0706.0312.

[45] M. C. M. Rentmeester, R. G. E. Timmermans and J. J. de Swart, Phys. Rev. C 67, 044001 (2003) arXiv:nuclth/0302080.

[46] R. P. Hildebrandt, H. W. Griehammer, T. R. Hemmert and B. Pasquini, Eur. Phys. J. A 20, 293 (2004) arXiv:nucl-th/0307070.

[47] A. I. Vainstein and V. I. Zakharov, Phys. Lett. B 72, 368 (1978).

[48] C. E. Carlson and F. Gross, Phys. Rev. Lett. 53, 127 (1984).

[49] V. Bernard, N. Kaiser, J. Kambor and U.-G. Meissner, Nucl. Phys. B 388, 287 (1992).

[50] V. Bernard, H. W. Fearing, T. R. Hemmert and U.-G. Meissner, Nucl. Phys. A 635, 121 (1998) arXiv:hep$\mathrm{ph} / 9801297$.

[51] K. Pachucki, Phys. Rev. A 53, 2093 (1996).

[52] J. Arrington, W. Melnitchouk and J. A. Tjon, Phys. Rev. C 76, 035205 (2007) arXiv:0707.1861.

[53] I. T. Lorenz, H.-W. Hammer and U.-G. Meissner, arXiv:1205:6628. 